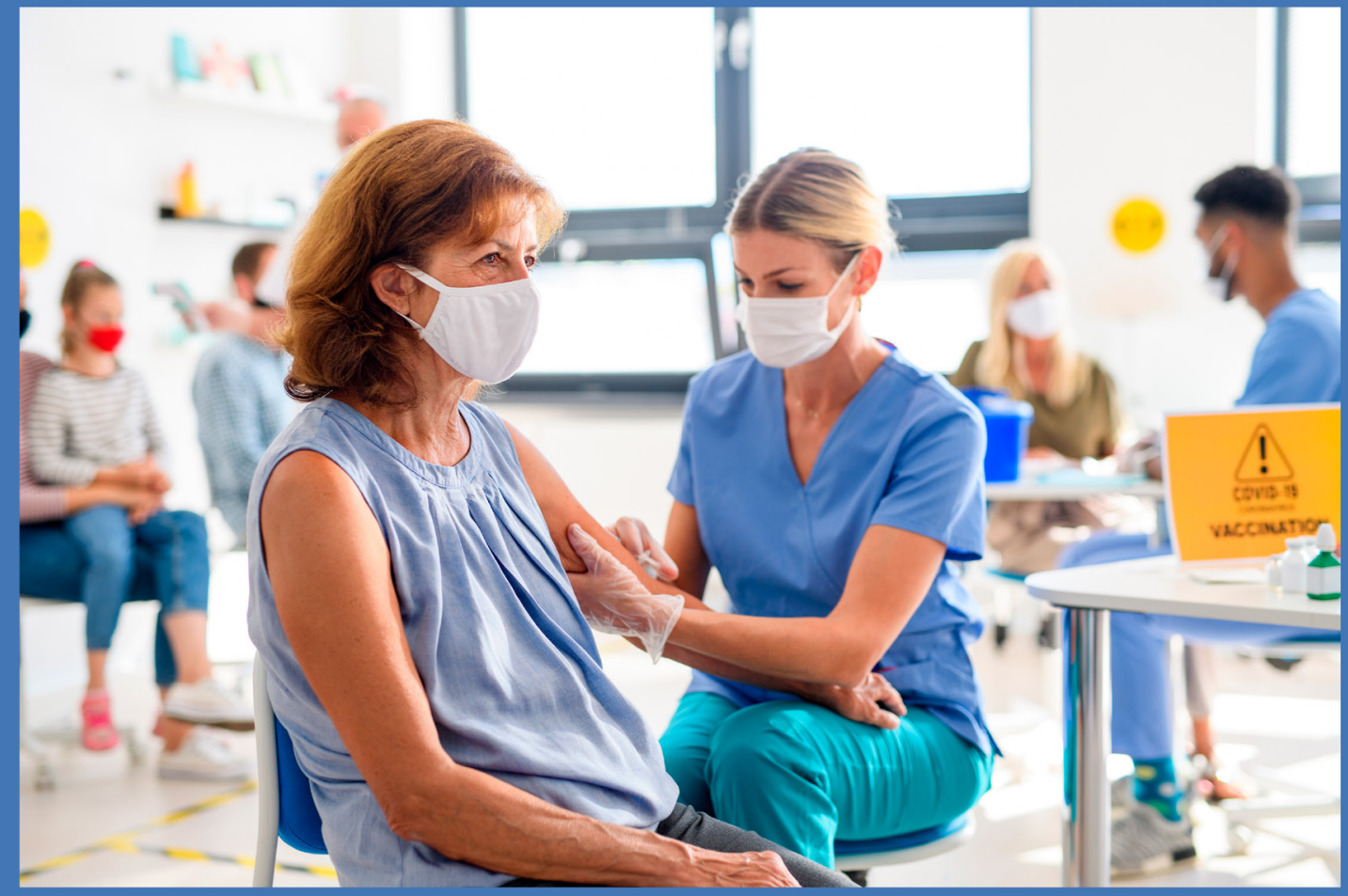

\title{
PRÁTICAS PREVENTIVAS E PRÁTICAS GURATIVAS NA MEDICINA 4
}

Benedito Rodrigues da Silva Neto (Organizador)

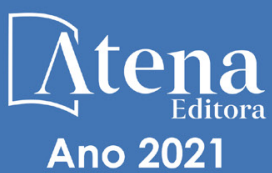




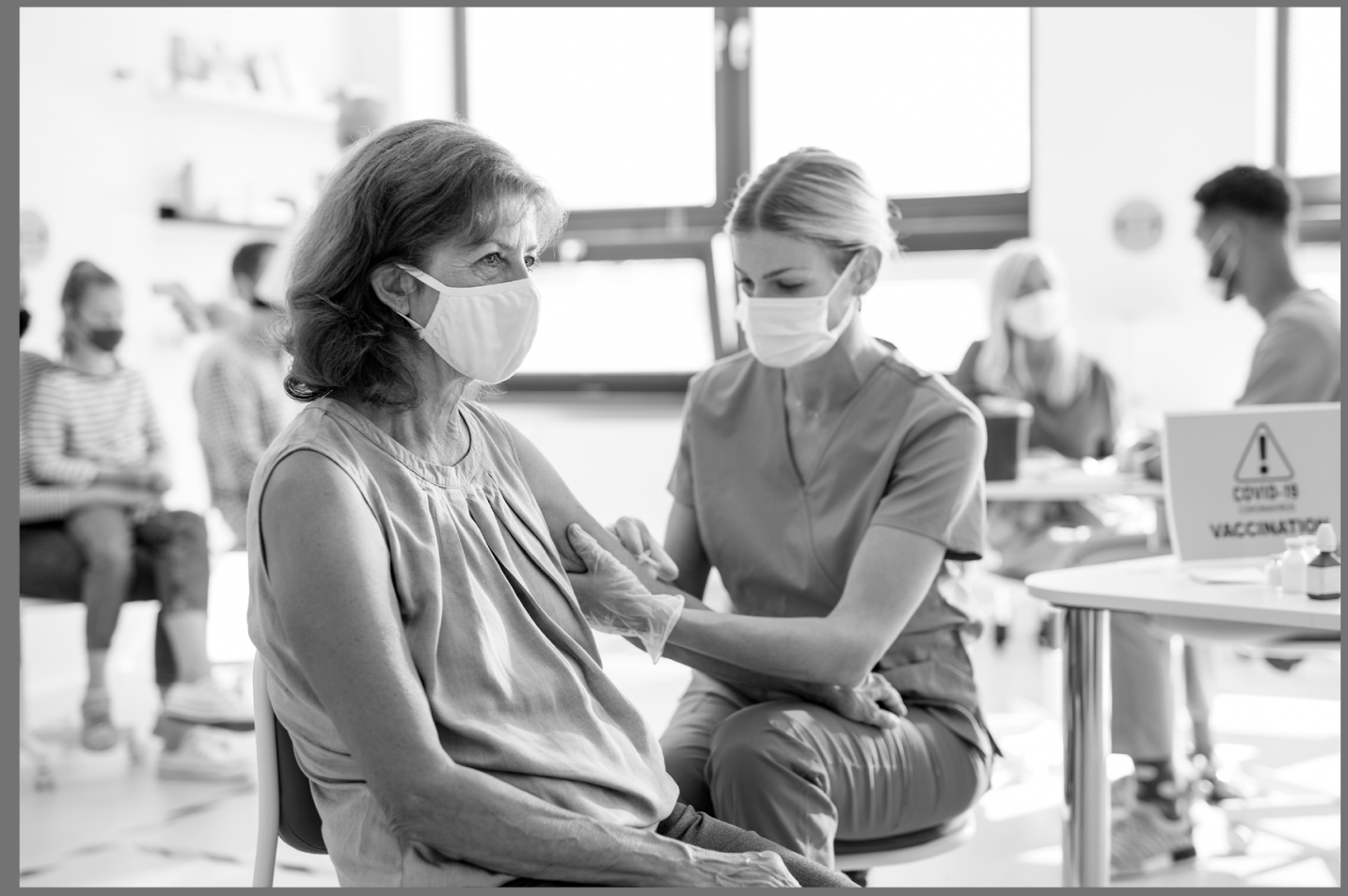

\section{PRÁTICAS PREVENTIVAS E PRÁTICAS GURATIVAS NA MEDICINA 4}

Benedito Rodrigues da Silva Neto (Organizador) 


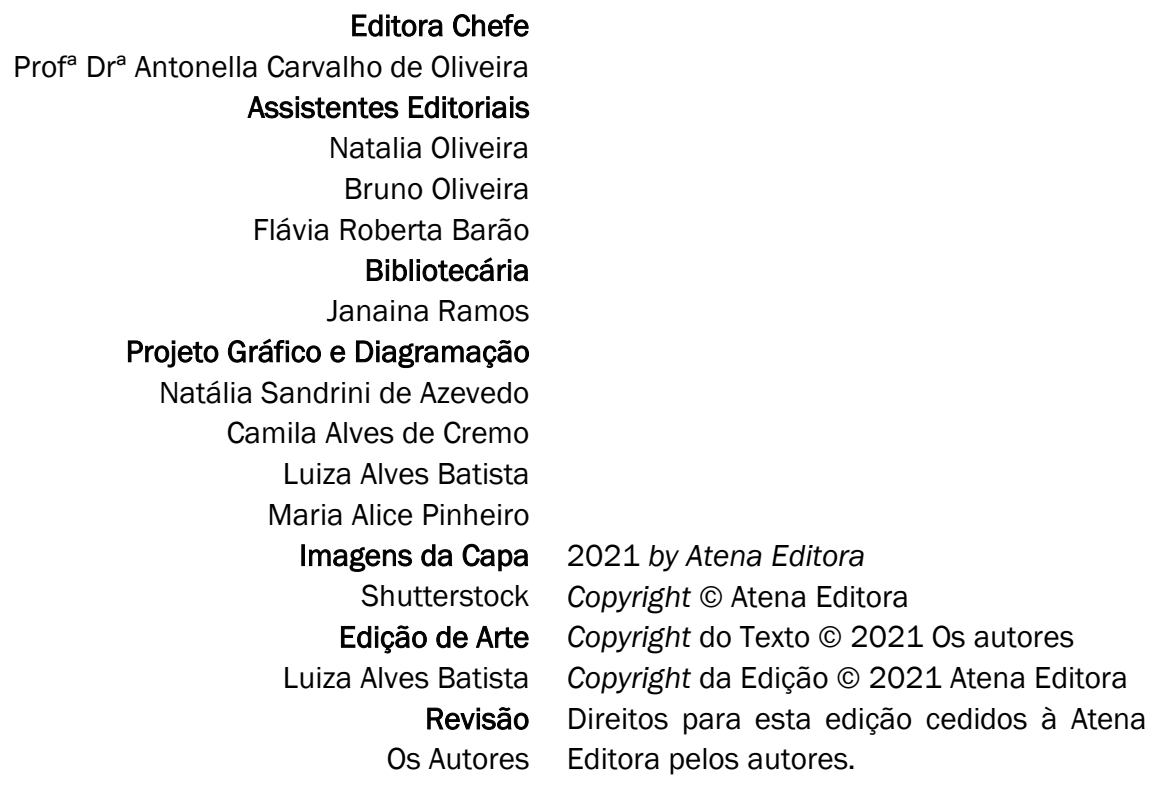

Todo o conteúdo deste livro está licenciado sob uma Licença de Atribuição Creative Commons. Atribuição-Não-ComercialNãoDerivativos 4.0 Internacional (CC BY-NC-ND 4.0).

O conteúdo dos artigos e seus dados em sua forma, correção e confiabilidade são de responsabilidade exclusiva dos autores, inclusive não representam necessariamente a posição oficial da Atena Editora. Permitido o download da obra e o compartilhamento desde que sejam atribuídos créditos aos autores, mas sem a possibilidade de alterá-la de nenhuma forma ou utilizá-la para fins comerciais.

Todos os manuscritos foram previamente submetidos à avaliação cega pelos pares, membros do Conselho Editorial desta Editora, tendo sido aprovados para a publicação com base em critérios de neutralidade e imparcialidade acadêmica.

A Atena Editora é comprometida em garantir a integridade editorial em todas as etapas do processo de publicação, evitando plágio, dados ou resultados fraudulentos e impedindo que interesses financeiros comprometam os padrões éticos da publicação. Situações suspeitas de má conduta científica serão investigadas sob o mais alto padrão de rigor acadêmico e ético.

\section{Conselho Editorial}

\section{Ciências Humanas e Sociais Aplicadas}

Prof. Dr. Alexandre Jose Schumacher - Instituto Federal de Educação, Ciência e Tecnologia do Paraná

Prof. Dr. Américo Junior Nunes da Silva - Universidade do Estado da Bahia Prof. Dr. Antonio Carlos Frasson - Universidade Tecnológica Federal do Paraná

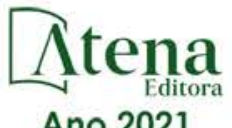


Prof. Dr. Antonio Gasparetto Júnior - Instituto Federal do Sudeste de Minas Gerais

Prof. Dr. Antonio Isidro-Filho - Universidade de Brasília

Prof. Dr. Carlos Antonio de Souza Moraes - Universidade Federal Fluminense

Prof. Dr. Crisóstomo Lima do Nascimento - Universidade Federal Fluminense

Prof $^{\mathrm{a}} \mathrm{Dr}^{\mathrm{a}}$ Cristina Gaio - Universidade de Lisboa

Prof. Dr. Daniel Richard Sant'Ana - Universidade de Brasília

Prof. Dr. Deyvison de Lima Oliveira - Universidade Federal de Rondônia

Prof $^{\mathrm{a}} \mathrm{Dr}^{\mathrm{a}}$ Dilma Antunes Silva - Universidade Federal de São Paulo

Prof. Dr. Edvaldo Antunes de Farias - Universidade Estácio de Sá

Prof. Dr. Elson Ferreira Costa - Universidade do Estado do Pará

Prof. Dr. Eloi Martins Senhora - Universidade Federal de Roraima

Prof. Dr. Gustavo Henrique Cepolini Ferreira - Universidade Estadual de Montes Claros

Prof $^{a}$ Dr $^{\mathrm{a}}$ Ivone Goulart Lopes - Istituto Internazionele delle Figlie de Maria Ausiliatrice

Prof. Dr. Jadson Correia de Oliveira - Universidade Católica do Salvador

Prof. Dr. Julio Candido de Meirelles Junior - Universidade Federal Fluminense

Prof $^{\mathrm{a}} \mathrm{Dr}^{\mathrm{a}}$ Lina Maria Gonçalves - Universidade Federal do Tocantins

Prof. Dr. Luis Ricardo Fernandes da Costa - Universidade Estadual de Montes Claros

Prof ${ }^{a}$ Dr $^{a}$ Natiéli Piovesan - Instituto Federal do Rio Grande do Norte

Prof. Dr. Marcelo Pereira da Silva - Pontifícia Universidade Católica de Campinas

Prof $^{\mathrm{a}}$ Dr $^{\mathrm{a}}$ Maria Luzia da Silva Santana - Universidade Federal de Mato Grosso do Sul

Prof $^{a}$ Dr $^{a}$ Paola Andressa Scortegagna - Universidade Estadual de Ponta Grossa

Prof $^{a}$ Dr $^{\mathrm{a}}$ Rita de Cássia da Silva Oliveira - Universidade Estadual de Ponta Grossa

Prof. Dr. Rui Maia Diamantino - Universidade Salvador

Prof. Dr. Urandi João Rodrigues Junior - Universidade Federal do Oeste do Pará

Prof $^{a}$ Dr $^{a}$ Vanessa Bordin Viera - Universidade Federal de Campina Grande

Prof. Dr. William Cleber Domingues Silva - Universidade Federal Rural do Rio de Janeiro

Prof. Dr. Willian Douglas Guilherme - Universidade Federal do Tocantins

\section{Ciências Agrárias e Multidisciplinar}

Prof. Dr. Alexandre Igor Azevedo Pereira - Instituto Federal Goiano

Prof $^{\mathrm{a}}$ Dr $^{\mathrm{a}}$ Carla Cristina Bauermann Brasil - Universidade Federal de Santa Maria

Prof. Dr. Antonio Pasqualetto - Pontifícia Universidade Católica de Goiás

Prof. Dr. Cleberton Correia Santos - Universidade Federal da Grande Dourados

Prof $^{a}$ Dr $^{a}$ Daiane Garabeli Trojan - Universidade Norte do Paraná

Prof $^{a}$ Dr $^{a}$ Diocléa Almeida Seabra Silva - Universidade Federal Rural da Amazônia

Prof. Dr. Écio Souza Diniz - Universidade Federal de Viçosa

Prof. Dr. Fábio Steiner - Universidade Estadual de Mato Grosso do Sul

Prof. Dr. Fágner Cavalcante Patrocínio dos Santos - Universidade Federal do Ceará

Prof ${ }^{a}$ Dr $^{a}$ Girlene Santos de Souza - Universidade Federal do Recôncavo da Bahia

Prof. Dr. Jael Soares Batista - Universidade Federal Rural do Semi-Árido

Prof. Dr. Júlio César Ribeiro - Universidade Federal Rural do Rio de Janeiro

Prof ${ }^{a}$ Dr $^{a}$ Lina Raquel Santos Araújo - Universidade Estadual do Ceará

Prof. Dr. Pedro Manuel Villa - Universidade Federal de Viçosa

Prof $^{a}$ Dr $^{\text {a }}$ Raissa Rachel Salustriano da Silva Matos - Universidade Federal do Maranhão

Prof. Dr. Ronilson Freitas de Souza - Universidade do Estado do Pará

Prof $^{a}$ Dr $^{a}$ Talita de Santos Matos - Universidade Federal Rural do Rio de Janeiro

Prof. Dr. Tiago da Silva Teófilo - Universidade Federal Rural do Semi-Árido

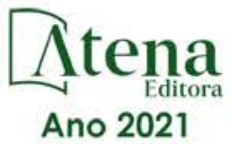


Prof. Dr. Valdemar Antonio Paffaro Junior - Universidade Federal de Alfenas

\section{Ciências Biológicas e da Saúde}

Prof. Dr. André Ribeiro da Silva - Universidade de Brasília

Prof $^{\mathrm{a}} \mathrm{Dr}^{\mathrm{a}}$ Anelise Levay Murari - Universidade Federal de Pelotas

Prof. Dr. Benedito Rodrigues da Silva Neto - Universidade Federal de Goiás

Prof $^{\mathrm{a}}$ Dr $^{\mathrm{a}}$ Débora Luana Ribeiro Pessoa - Universidade Federal do Maranhão

Prof. Dr. Douglas Siqueira de Almeida Chaves - Universidade Federal Rural do Rio de Janeiro

Prof. Dr. Edson da Silva - Universidade Federal dos Vales do Jequitinhonha e Mucuri

Prof $^{a}$ Dr $^{a}$ Elizabeth Cordeiro Fernandes - Faculdade Integrada Medicina

Prof $^{\mathrm{a}} \mathrm{Dr}^{\mathrm{a}}$ Eleuza Rodrigues Machado - Faculdade Anhanguera de Brasília

Prof $^{\mathrm{a}} \mathrm{Dr}^{\mathrm{a}}$ Elane Schwinden Prudêncio - Universidade Federal de Santa Catarina

Prof $^{a}$ Dr $^{a}$ Eysler Gonçalves Maia Brasil - Universidade da Integração Internacional da Lusofonia

Afro-Brasileira

Prof. Dr. Ferlando Lima Santos - Universidade Federal do Recôncavo da Bahia

Prof. Dr. Fernando Mendes - Instituto Politécnico de Coimbra - Escola Superior de Saúde de Coimbra

Prof $^{\mathrm{a}} \mathrm{Dr}^{\mathrm{a}}$ Gabriela Vieira do Amaral - Universidade de Vassouras

Prof. Dr. Gianfábio Pimentel Franco - Universidade Federal de Santa Maria

Prof. Dr. Helio Franklin Rodrigues de Almeida - Universidade Federal de Rondônia

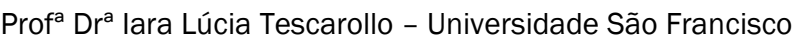

Prof. Dr. Igor Luiz Vieira de Lima Santos - Universidade Federal de Campina Grande

Prof. Dr. Jefferson Thiago Souza - Universidade Estadual do Ceará

Prof. Dr. Jesus Rodrigues Lemos - Universidade Federal do Piauí

Prof. Dr. Jônatas de França Barros - Universidade Federal do Rio Grande do Norte

Prof. Dr. José Max Barbosa de Oliveira Junior - Universidade Federal do Oeste do Pará

Prof. Dr. Luís Paulo Souza e Souza - Universidade Federal do Amazonas

Prof $^{a}$ Dr $^{a}$ Magnólia de Araújo Campos - Universidade Federal de Campina Grande

Prof. Dr. Marcus Fernando da Silva Praxedes - Universidade Federal do Recôncavo da Bahia

Prof $^{a}$ Dr $^{a}$ Maria Tatiane Gonçalves Sá - Universidade do Estado do Pará

Prof $^{a}$ Dr $^{\mathrm{a}}$ Mylena Andréa Oliveira Torres - Universidade Ceuma

Prof $^{a}$ Dr $^{a}$ Natiéli Piovesan - Instituto Federacl do Rio Grande do Norte

Prof. Dr. Paulo Inada - Universidade Estadual de Maringá

Prof. Dr. Rafael Henrique Silva - Hospital Universitário da Universidade Federal da Grande

Dourados

Prof $^{\mathrm{a}} \mathrm{Dr}^{\mathrm{a}}$ Regiane Luz Carvalho - Centro Universitário das Faculdades Associadas de Ensino

Prof $^{a}$ Dr $^{a}$ Renata Mendes de Freitas - Universidade Federal de Juiz de Fora

Prof $^{\mathrm{a}} \mathrm{Dr}^{\mathrm{a}}$ Vanessa Lima Gonçalves - Universidade Estadual de Ponta Grossa

Prof $^{a}$ Dr $^{a}$ Vanessa Bordin Viera - Universidade Federal de Campina Grande

\section{Ciências Exatas e da Terra e Engenharias}

Prof. Dr. Adélio Alcino Sampaio Castro Machado - Universidade do Porto

Prof. Dr. Carlos Eduardo Sanches de Andrade - Universidade Federal de Goiás

Prof $^{a}$ Dr $^{\mathrm{a}}$ Carmen Lúcia Voigt - Universidade Norte do Paraná

Prof. Dr. Cleiseano Emanuel da Silva Paniagua - Instituto Federal de Educação, Ciência e Tecnologia de Goiás

Prof. Dr. Douglas Gonçalves da Silva - Universidade Estadual do Sudoeste da Bahia

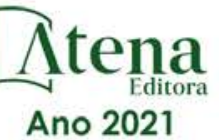


Prof. Dr. Eloi Rufato Junior - Universidade Tecnológica Federal do Paraná

Prof $^{\mathrm{a}}$ Dr $^{\mathrm{a}}$ Érica de Melo Azevedo - Instituto Federal do Rio de Janeiro

Prof. Dr. Fabrício Menezes Ramos - Instituto Federal do Pará

Prof ${ }^{a}$ Dra. Jéssica Verger Nardeli - Universidade Estadual Paulista Júlio de Mesquita Filho

Prof. Dr. Juliano Carlo Rufino de Freitas - Universidade Federal de Campina Grande

Prof $^{a}$ Dr $^{a}$ Luciana do Nascimento Mendes - Instituto Federal de Educação, Ciência e Tecnologia do Rio Grande do Norte

Prof. Dr. Marcelo Marques - Universidade Estadual de Maringá

Prof. Dr. Marco Aurélio Kistemann Junior - Universidade Federal de Juiz de Fora

Prof $^{\mathrm{a}} \mathrm{Dr}^{\mathrm{a}}$ Neiva Maria de Almeida - Universidade Federal da Paraíba

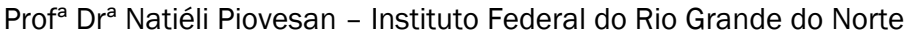

Prof $^{a}$ Dr $^{\text {a }}$ Priscila Tessmer Scaglioni - Universidade Federal de Pelotas

Prof. Dr. Takeshy Tachizawa - Faculdade de Campo Limpo Paulista

\section{Linguística, Letras e Artes}

Prof $^{a}$ Dra $^{a}$ Adriana Demite Stephani - Universidade Federal do Tocantins

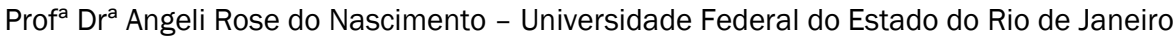

Prof $^{a}$ Dr $^{a}$ Carolina Fernandes da Silva Mandaji - Universidade Tecnológica Federal do Paraná

Prof $^{\mathrm{a}} \mathrm{Dr}^{\mathrm{a}}$ Denise Rocha - Universidade Federal do Ceará

Prof. Dr. Fabiano Tadeu Grazioli - Universidade Regional Integrada do Alto Uruguai e das Missões

Prof. Dr. Gilmei Fleck - Universidade Estadual do Oeste do Paraná

Prof $^{a}$ Dr $^{a}$ Keyla Christina Almeida Portela - Instituto Federal de Educação, Ciência e Tecnologia do Paraná

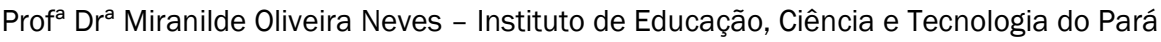

Prof $^{\mathrm{a}} \mathrm{Dr}^{\mathrm{a}}$ Sandra Regina Gardacho Pietrobon - Universidade Estadual do Centro-Oeste

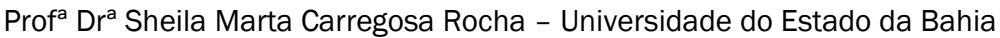

\section{Conselho Técnico Científico}

Prof. Me. Abrãao Carvalho Nogueira - Universidade Federal do Espírito Santo

Prof. Me. Adalberto Zorzo - Centro Estadual de Educação Tecnológica Paula Souza

Prof. Dr. Adaylson Wagner Sousa de Vasconcelos - Ordem dos Advogados do Brasil/Seccional Paraíba

Prof. Dr. Adilson Tadeu Basquerote Silva - Universidade para o Desenvolvimento do Alto Vale do Itajaí

Prof. Dr. Alex Luis dos Santos - Universidade Federal de Minas Gerais

Prof. Me. Alexsandro Teixeira Ribeiro - Centro Universitário Internacional

Prof $^{\mathrm{a}} \mathrm{Ma}$. Aline Ferreira Antunes - Universidade Federal de Goiás

Prof. Me. André Flávio Gonçalves Silva - Universidade Federal do Maranhão

Prof ${ }^{a}$ Ma. Andréa Cristina Marques de Araújo - Universidade Fernando Pessoa

Prof $^{\text {a }}$ Dr $^{\text {a }}$ Andreza Lopes - Instituto de Pesquisa e Desenvolvimento Acadêmico

Prof $^{a}$ Dr $^{a}$ Andrezza Miguel da Silva - Faculdade da Amazônia

Profa Ma. Anelisa Mota Gregoleti - Universidade Estadual de Maringá

Prof $^{a}$ Ma. Anne Karynne da Silva Barbosa - Universidade Federal do Maranhão

Prof. Dr. Antonio Hot Pereira de Faria - Polícia Militar de Minas Gerais

Prof. Me. Armando Dias Duarte - Universidade Federal de Pernambuco

Prof $^{a}$ Ma. Bianca Camargo Martins - UniCesumar

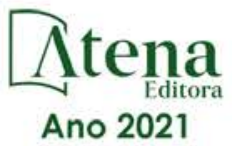


Prof $^{a}$ Ma. Carolina Shimomura Nanya - Universidade Federal de São Carlos

Prof. Me. Carlos Antônio dos Santos - Universidade Federal Rural do Rio de Janeiro

Prof. Me. Christopher Smith Bignardi Neves - Universidade Federal do Paraná

Prof. Ma. Cláudia de Araújo Marques - Faculdade de Música do Espírito Santo

Prof $^{a}$ Dr $^{\text {a }}$ Cláudia Taís Siqueira Cagliari - Centro Universitário Dinâmica das Cataratas

Prof. Me. Clécio Danilo Dias da Silva - Universidade Federal do Rio Grande do Norte

Prof. Me. Daniel da Silva Miranda - Universidade Federal do Pará

Prof $^{a}$ Ma. Daniela da Silva Rodrigues - Universidade de Brasília

Prof $^{a}$ Ma. Daniela Remião de Macedo - Universidade de Lisboa

Prof ${ }^{a}$ Ma. Dayane de Melo Barros - Universidade Federal de Pernambuco

Prof. Me. Douglas Santos Mezacas - Universidade Estadual de Goiás

Prof. Me. Edevaldo de Castro Monteiro - Embrapa Agrobiologia

Prof. Me. Eduardo Gomes de Oliveira - Faculdades Unificadas Doctum de Cataguases

Prof. Me. Eduardo Henrique Ferreira - Faculdade Pitágoras de Londrina

Prof. Dr. Edwaldo Costa - Marinha do Brasil

Prof. Me. Eliel Constantino da Silva - Universidade Estadual Paulista Júlio de Mesquita

Prof. Me. Ernane Rosa Martins - Instituto Federal de Educação, Ciência e Tecnologia de Goiás

Prof. Me. Euvaldo de Sousa Costa Junior - Prefeitura Municipal de São João do Piauí

Prof. Dr. Everaldo dos Santos Mendes - Instituto Edith Theresa Hedwing Stein

Prof. Me. Ezequiel Martins Ferreira - Universidade Federal de Goiás

Prof ${ }^{a}$ Ma. Fabiana Coelho Couto Rocha Corrêa - Centro Universitário Estácio Juiz de Fora

Prof. Me. Fabiano Eloy Atílio Batista - Universidade Federal de Viçosa

Prof. Me. Felipe da Costa Negrão - Universidade Federal do Amazonas

Prof. Me. Francisco Odécio Sales - Instituto Federal do Ceará

Prof ${ }^{a}$ Dr $^{\mathrm{a}}$ Germana Ponce de Leon Ramírez - Centro Universitário Adventista de São Paulo

Prof. Me. Gevair Campos - Instituto Mineiro de Agropecuária

Prof. Me. Givanildo de Oliveira Santos - Secretaria da Educação de Goiás

Prof. Dr. Guilherme Renato Gomes - Universidade Norte do Paraná

Prof. Me. Gustavo Krahl - Universidade do Oeste de Santa Catarina

Prof. Me. Helton Rangel Coutinho Junior - Tribunal de Justiça do Estado do Rio de Janeiro

Prof $^{\mathrm{a}} \mathrm{Ma}$. Isabelle Cerqueira Sousa - Universidade de Fortaleza

Prof $^{a}$ Ma. Jaqueline Oliveira Rezende - Universidade Federal de Uberlândia

Prof. Me. Javier Antonio Albornoz - University of Miami and Miami Dade College

Prof. Me. Jhonatan da Silva Lima - Universidade Federal do Pará

Prof. Dr. José Carlos da Silva Mendes - Instituto de Psicologia Cognitiva, Desenvolvimento Humano e Social

Prof. Me. Jose Elyton Batista dos Santos - Universidade Federal de Sergipe

Prof. Me. José Luiz Leonardo de Araujo Pimenta - Instituto Nacional de Investigación Agropecuaria Uruguay

Prof. Me. José Messias Ribeiro Júnior - Instituto Federal de Educação Tecnológica de Pernambuco

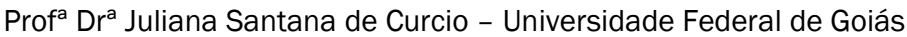

Prof ${ }^{a}$ Ma. Juliana Thaisa Rodrigues Pacheco - Universidade Estadual de Ponta Grossa

Prof $^{a}$ Dr $^{a}$ Kamilly Souza do Vale - Núcleo de Pesquisas Fenomenológicas/UFPA

Prof. Dr. Kárpio Márcio de Siqueira - Universidade do Estado da Bahia

Prof $^{a}$ Dr $^{a}$ Karina de Araújo Dias - Prefeitura Municipal de Florianópolis

Prof. Dr. Lázaro Castro Silva Nascimento - Laboratório de Fenomenologia \& Subjetividade/UFPR

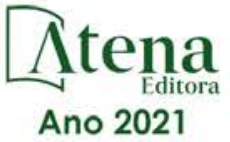


Prof. Me. Leonardo Tullio - Universidade Estadual de Ponta Grossa

Prof ${ }^{a}$ Ma. Lilian Coelho de Freitas - Instituto Federal do Pará

Prof $^{\text {a }}$ Ma. Liliani Aparecida Sereno Fontes de Medeiros - Consórcio CEDERJ

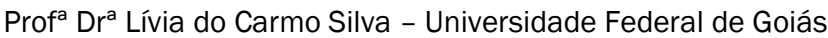

Prof. Dr. Lucio Marques Vieira Souza - Secretaria de Estado da Educação, do Esporte e da Cultura de Sergipe

Prof. Dr. Luan Vinicius Bernardelli - Universidade Estadual do Paraná

Prof $^{\mathrm{a}} \mathrm{Ma}$. Luana Ferreira dos Santos - Universidade Estadual de Santa Cruz

Prof $^{a}$ Ma. Luana Vieira Toledo - Universidade Federal de Viçosa

Prof. Me. Luis Henrique Almeida Castro - Universidade Federal da Grande Dourados

Prof $^{a}$ Ma. Luma Sarai de Oliveira - Universidade Estadual de Campinas

Prof. Dr. Michel da Costa - Universidade Metropolitana de Santos

Prof. Me. Marcelo da Fonseca Ferreira da Silva - Governo do Estado do Espírito Santo

Prof. Dr. Marcelo Máximo Purificação - Fundação Integrada Municipal de Ensino Superior

Prof. Me. Marcos Aurelio Alves e Silva - Instituto Federal de Educação, Ciência e Tecnologia de São Paulo

Prof ${ }^{a}$ Ma. Maria Elanny Damasceno Silva - Universidade Federal do Ceará

Prof ${ }^{a}$ Ma. Marileila Marques Toledo - Universidade Federal dos Vales do Jequitinhonha e Mucuri

Prof. Me. Pedro Panhoca da Silva - Universidade Presbiteriana Mackenzie

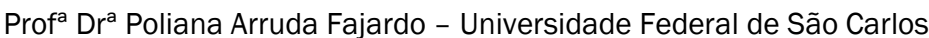

Prof. Me. Ricardo Sérgio da Silva - Universidade Federal de Pernambuco

Prof. Me. Renato Faria da Gama - Instituto Gama - Medicina Personalizada e Integrativa

Prof ${ }^{a}$ Ma. Renata Luciane Polsaque Young Blood - UniSecal

Prof. Me. Robson Lucas Soares da Silva - Universidade Federal da Paraíba

Prof. Me. Sebastião André Barbosa Junior - Universidade Federal Rural de Pernambuco

Prof $^{a}$ Ma. Silene Ribeiro Miranda Barbosa - Consultoria Brasileira de Ensino, Pesquisa e Extensão

Prof $^{a}$ Ma. Solange Aparecida de Souza Monteiro - Instituto Federal de São Paulo

Prof ${ }^{a}$ Ma. Taiane Aparecida Ribeiro Nepomoceno - Universidade Estadual do Oeste do Paraná

Prof. Me. Tallys Newton Fernandes de Matos - Faculdade Regional Jaguaribana

Prof $^{a}$ Ma. Thatianny Jasmine Castro Martins de Carvalho - Universidade Federal do Piauí

Prof. Me. Tiago Silvio Dedoné - Colégio ECEL Positivo

Prof. Dr. Welleson Feitosa Gazel - Universidade Paulista

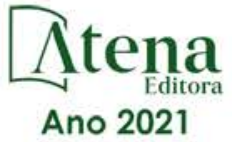


Editora Chefe: Prof $^{\mathrm{a}} \mathrm{Dr}^{\mathrm{a}}$ Antonella Carvalho de Oliveira

Bibliotecária: Janaina Ramos

Diagramação: Camila Alves de Cremo

Correção: Giovanna Sandrini de Azevedo

Edição de Arte: Luiza Alves Batista

Revisão: Os Autores

Organizador: Benedito Rodrigues da Silva Neto

\begin{tabular}{|c|c|}
\hline \multicolumn{2}{|r|}{ Dados Internacionais de Catalogação na Publicação (CIP) } \\
\hline & $\begin{array}{l}\text { Práticas preventivas e práticas curativas na medicina } 4 \text { / } \\
\text { Organizador Benedito Rodrigues da Silva Neto. - Ponta } \\
\text { Grossa - PR: Atena, } 2021 . \\
\text { Formato: PDF } \\
\text { Requisitos de sistema: Adobe Acrobat Reader } \\
\text { Modo de acesso: World Wide Web } \\
\text { Inclui bibliografia } \\
\text { ISBN 978-65-5706-863-2 } \\
\text { DOI 10.22533/at.ed.632210103 } \\
\text { 1. Medicina. 2. Saúde. I. Silva Neto, Benedito } \\
\text { Rodrigues da (Organizador). II. Título. }\end{array}$ \\
\hline & borado por Bibliotecária Janaina Ramos - CRB-8/9166 \\
\hline
\end{tabular}

Atena Editora

Ponta Grossa - Paraná - Brasil Telefone: +55 (42) 3323-5493 www.atenaeditora.com.br contato@atenaeditora.com.br 


\section{DECLARAÇÃO DOS AUTORES}

Os autores desta obra: 1. Atestam não possuir qualquer interesse comercial que constitua um conflito de interesses em relação ao artigo científico publicado; 2. Declaram que participaram ativamente da construção dos respectivos manuscritos, preferencialmente na: a) Concepção do estudo, e/ou aquisição de dados, e/ou análise e interpretação de dados; b) Elaboração do artigo ou revisão com vistas a tornar o material intelectualmente relevante; c) Aprovação final do manuscrito para submissão.; 3. Certificam que os artigos científicos publicados estão completamente isentos de dados e/ou resultados fraudulentos; 4. Confirmam a citação e a referência correta de todos os dados e de interpretações de dados de outras pesquisas; 5 . Reconhecem terem informado todas as fontes de financiamento recebidas para a consecução da pesquisa. 


\section{APRESENTAÇÃO}

A práticas preventivas e práticas curativas, que por muito tempo andavam separadas e aplicadas a momentos distintos dos processos de saúde e doença dos indivíduos, cada vez mais tem adquirido um aspecto complementar, principalmente quando consideramos a Saúde Pública como uma missão, no sentido de viabilizar um bem social comum garantindo as condições de saúde para a população.

Esse modo de pensar a medicina e a saúde coletiva tem orientado as mudanças nas políticas de saúde no Brasil, mais precisamente a partir da Constituição de 1988, onde o princípio do direito universal à atenção à saúde se fundamentou em diretrizes para a descentralização e integralidade das ações, e principalmente na participação comunitária.

A Medicina preventiva por conceito está voltada fundamentalmente aos cuidados rotineiros e antecipados, contemplando a adesão aos programas de vacinação, a realização de check-ups e exames periódicos, a prática de atividade física regular e iniciativas relacionadas à saúde mental, como a prática de meditação e psicoterapias. Já a Medicina curativa é aquela direcionada à cura de enfermidades e/ou tratamento de sintomas, evitando o agravamento e aparecimento de complicações. As estratégias são muitas e variadas, de acordo com a doença a ser combatida, podendo englobar tratamentos medicamentosos, terapias, intervenções cirúrgicas, etc.

Baseados nos conceitos, e no caminhar lado-a-lado dessas duas abordagens, propomos com esta obra oferecer ao leitor material de qualidade fundamentado produções acadêmicas, desenvolvendo os principais conceitos e discutindo diferentes métodos relacionados à temática central dos quatro volumes iniciais.

Finalmente destacamos a importância da Atena Editora como mecanismo de viabilização dos dados através de uma literatura, rigorosamente avaliada e fundamentada.

Desfrute ao máximo desta literatura! 


\section{CAPÍTULO 15 \\ INTELIGÊNCIA ARTIFICIAL APLICADA À OTIMIZAÇÃO DO DIAGNÓSTICO POR IMAGEM}

Data de aceite: 26/02/2021

Data de submissão: 02/01/2021

\section{Natanael Matos Santos}

Quixabeira - Bahia

http://lattes.cnpq.br/6054994219445222

\section{Cassio Fabian Sarquis de Campos}

Universidade do Oeste Paulista Faculdade de Ciências da saúde e engenharia

Presidente Prudente - São Paulo http://lattes.cnpq.br/9358644315119346

RESUMO: O uso da tecnologia para o diagnóstico por imagem tem crescido e otimizado a qualidade dos diagnósticos, velocidade dos exames e diminuindo o nível de dose para os pacientes. Este trabalho foi desenvolvido utilizando o padrão de estudo exploratório, por meio de pesquisa bibliográfica de materiais já produzidos, e tem por objetivo verificar a presença da Inteligência Artificial (IA) aplicada à radiologia como meio de otimização do diagnóstico por imagem e despertar os profissionais da área sobre a evolução da tecnologia para obtenção de um melhor diagnóstico médico, concluindo que, futuramente, pode ser exigido uma adaptação dos mesmos para sua inserção ou permanência no mercado de trabalho, mostrando a importância da IA na radiologia de maneira que os profissionais da saúde devem se desenvolver nesta área, tendo em vista que a sua aplicação pode trazer grandes benefícios à humanidade.

PALAVRAS-CHAVE: Radiologia, inteligência artificial, diagnóstico por imagem, covid-19, software de diagnóstico

\section{ARTIFICIAL INTELLIGENCE APPLIED TO} OPTIMIZATION OF IMAGING DIAGNOSIS

ABSTRACT: The use of technology for imaging has grown and optimized the quality of the diagnostics, speed of the examinations, and the level of dose for the patients. This work was developed using the exploratory study standard, through a bibliographical research of materials already produced, and aims to verify the presence of Artificial Intelligence (IA) applied to radiology as a means of optimizing the imaging diagnosis and awakening the professionals of the area on the evolution of technology to obtain a better medical diagnosis, concluding that, in the future, it may be required to adapt them to their insertion or permanence in the labor market, showing the importance of $\mathrm{Al}$ in radiology so that health professionals must develop in this area, since its application can bring great benefits to humanity. KEYWORDS: Radiology, artificial intelligence, diagnostic imaging, covid-19, diagnostic software.

\section{INTRODUÇÃO}

Ao se pesquisar o conceito de inteligência artificial, depara-se com definições bastante interessantes as quais mencionam que o termo "Artificial Intelligence" (A.I. - I.A. em português) foi usado pela primeira vez em 1956 por McCarthy (e desenvolvido por grandes pesquisadores como Marvin Minky e Herbert Simon) e corresponde em dizer que esta 
ferramenta nada mais é do que um conjunto de teorias e técnicas empregadas com a finalidade de desenvolver máquinas capazes de simular a inteligência humana. 1, 2, 3, 4, 5, 6, 7

A IA se refere ao ramo da ciência da computação dedicado ao desenvolvimento de algoritmos de computador para realizar tarefas tradicionalmente associadas à capacidade cognitiva humana, como a capacidade de aprender e resolver problemas. É uma abordagem interdisciplinar usando princípios e dispositivos de computação, matemática, lógica, mecânica e até mesmo biologia para resolver problemas da compreensão, modelagem e replicação da inteligência cognitiva. ${ }^{4,6,7,8}$

O aprendizado natural ${ }^{4}$ é um conceito de grande importância para que se possa partir para a construção de sistemas inteligentes dotados da capacidade de aprendizado. Assim, a capacidade de aprender está ligada diretamente aos seguintes itens:

- Adaptação: um sistema biológico ou artificial que não seja capaz de evoluir ou de mudar seu comportamento diante de novas situações que lhe são apresentadas não constitui um sistema inteligente; ${ }^{9}$

- Correção de erros: um sistema inteligente deve modificar seu comportamento atual de modo que satisfaça alguma exigência;

- Otimização: melhoria da performance do sistema como um todo atendendo sempre as condições do projeto. ${ }^{9}$

- Bases de dados: O sistema deve ser capaz de armazenar uma grande quantidade de informações para serem usadas ou empregadas segundo a necessidade do processo. ${ }^{9}$

Existe a necessidade de que a IA acresça novos conhecimentos para se auto aprimorar através da coleta de padrões de dados que não foram previamente programados. Esta capacidade de aprimoramento de aprendizado de máquina é conhecida como "Machine Learning". Isto possibilita que computadores realizem procedimentos complexos com conhecimento mais ampliado. ${ }^{9}$

"Machine Learning" é uma área da Inteligência Artificial que dá aos computadores a capacidade humana de decifrar e entender padrões por meio de dados previamente adicionados ao sistema. Processando dados repetidamente, ele se torna capaz de criar novos dados através do cruzamento dos padrões de informações obtidas anteriormente. Esse aprimoramento veloz e especificamente aplicado traz um enorme avanço para a IA tornando possível realizar tarefas que não eram possíveis antes. Tal avanço é o motivo que se leva à exploração de tal área. ${ }^{8,10}$

É difícil para o ser humano programar as máquinas, já que nem sempre é possível descrever a resolução de problemas, ou diagnósticos, realizados intuitivamente ou por aspectos que, geralmente, não parecem estar correlacionados. Mas o que leva tempo e muito esforço para o ser humano, não seria um processo tão desgastante e lento se as máquinas fossem programadas para isso, como por exemplo, emitir um diagnóstico médico 
a partir de uma imagem. ${ }^{9}$

A radiologia é uma das áreas que mais tem se destacado no uso da inteligência artificial. Os raios-x de tórax são os exames de imagem médica mais utilizados, chegando a cerca de 2 bilhões de exames realizados no mundo anualmente. As redes neurais convolucionais profundas (Deep CNNs) têm diagnosticado patologias em radiografias tão bem quanto muitos radiologistas que fizeram parte do teste de comparação de diagnóstico, para avaliar se a IA realmente emitiria os diagnósticos à altura dos próprios radiologistas. ${ }^{11}$

Com a radiologia, os avanços não são diferentes aos experimentados por outras áreas, uma vez que, tem-se evoluções desde 1985, quando os Raios-X foram descobertos, até o presente dia, com as mais variadas técnicas de radiodiagnósticos digitais. A radiologia digital é o ramo do diagnóstico médico que utiliza diversas metodologias aplicadas por sistemas computacionais a fim de adquirir, transferir, armazenar e tratar as imagens radiográficas adquiridas a partir de quase todas as modalidades. Esta área vem experimentando grandes mudanças graças aos avanços tecnológicos e os métodos de imagem que surgiram após as radiografias convencionais serem substituídas por radiografias digitais, coloridas e de reconstruções multiplanares em $2 \mathrm{D}$ e $3 \mathrm{D}$, o que vem possibilitando diagnósticos muito mais precisos para os pacientes e diminuições significativas de erros nos achados radiográficos. $12,13,14$

Com essa grande variação de imagens, as Redes Neurais Convolucionais (CNNs) se tornaram uma grande alavanca da IA por sua grande capacidade de reconhecimento e processamento de imagens. As CNNs possuem várias camadas de processamento de informações que analisarão as imagens, principalmente reconhecendo padrões, o que requer uma base de dados previamente processada para comparações. São compostas de "neurônios" que se otimizam automaticamente por "Machine Learning", a qual cada camada de neurônio processará, de acordo aos algoritmos programados, a largura, altura e a densidade dos pixels. ${ }^{8,14,15,16,17}$

\section{INTELIGÊNCIA ARTIFICIAL APLICADA À RADIOLOGIA}

As FCNs (Fully convolutional network - Rede totalmente convolucional) tornaram viáveis o treinamento de modelos para linguagem de pixels com segmentação de ponta a ponta. Isso possibilitou, com ajuda de boas memórias GPUs, o melhoramento da reconstrução de imagens 3D dos órgãos que se encontram no abdome e tórax. Este exemplo de modelo alcança performances de ponta em segmentação automatizada de múltiplos órgãos de TC abdominal com média de $90 \%$ dos dados testados em todos os órgãos visados. ${ }^{18}$

Pesquisadores da Universidade de Ciência e Tecnologia da Noruega aplicaram um filtro para detectar e localizar núcleos em imagens médicas. Para isso implementaram e usaram um método de última geração chamada "Mask R-CNN", que é uma rede neural 
convolucional desenvolvida para análise segmentada de imagens, que se mostrou capaz de detectar células troncos em imagens médicas. ${ }^{19}$

\section{DEEP LEARNING (DL)}

Para identificar núcleos celulares e proteínas fluorescentes, os agentes de coloração têm sido amplamente utilizados. Contudo, agentes exógenos previnem, inevitavelmente, a visualização a longo prazo de células vivas e a análise rápida, e até mesmo interferem em condições fisiológicas intrínsecas. Pesquisadores da Coreia do Sul propuseram um método de segmentação livre de rótulos de núcleos celulares em imagens de tomografia de difração óptica, utilizando uma estrutura de aprendizagem profunda (Deep Learning ou DL). O método proposto foi aplicado para a segmentação precisa do núcleo da célula em imagens sem rótulos em duas, três ou quatro dimensões e visa trazer aplicações biomédicas amplas e imediatas aos quadros clínicos analisados por imagens. ${ }^{19,20}$

Os pesquisadores Rajpurkar $\mathrm{P}$, et al. desenvolveram um algoritmo de aprendizagem profunda para detectar patologias nas radiografias de tórax e assim compará-las com as patologias achadas pelos radiologistas. Esta rede neural foi chamada de CheXNeXt, programada para detectar 14 tipos diferentes de patologias na radiografia convencional do tórax, e descobriram que esse modelo de DL detecta tão bem as diferentes patologias presentes nas imagens quanto os próprios radiologistas. ${ }^{21}$

Cientistas japoneses desenvolveram uma IA usando "Deep Learning" para melhorar a imagem adquirida por tomografia computadorizada (TC) e ressonância magnética (RM). Eles categorizaram as técnicas para melhorar a qualidade da imagem como "redução de ruído e artefato", "super-resolução" e "aquisição e reconstrução de imagem". A redução de ruídos e artefatos ajudaram a diminuir a exposição à radiação nos exames de TC e a encurtar o tempo de varredura da RM (Ressonância Magnética). A técnica de superresolução pode otimizar o diagnóstico, melhorando a resolução das imagens espessas e encurtar o tempo de aquisição de imagens necessário para exames de RM, o que é muito benéfico aos pacientes, independente da patologia. ${ }^{22}$

\section{REDES NEURAIS CONVOLUCIONAIS (CNNS) COM DEEP LEARNING}

Alguns pesquisadores usaram redes neurais convolucionais (CNNs) para detectar indícios de pneumonia presentes nas radiografias de tórax, para isso usaram uma base de dados com 158.323 radiografias de tórax de 3 instituições distintas e descobriram que a CNN não só detecta achados específicos da doença na imagem, como também tem capacidade de processar informações confusas. ${ }^{16,17,23,24,25}$

Fang Liu e seus colegas desenvolveram um modelo de rede neural de aprendizado profundo usando imagens de tomografia computadorizada a fim de minimizar as falhas das imagens adquiridas por PET-MRI (Tomografia por Emissão de Positrões - Imagem por 
Ressonância Magnética) e melhorá-las, já que a ressonância magnética não adquire boas imagens de estruturas ósseas. Usaram 30 imagens tridimensionais para treinar o modelo, e assim, foi avaliado em 10 pacientes, comparando as imagens geradas pelo modelo com as imagens adquiridas. Como resultado, as imagens foram reconstruídas com menos de $1 \%$ de falha, ao contrário das abordagens convencionais de reconstrução de imagem. ${ }^{26}$

Um método de aprendizagem profunda (Deep Learning) com uma rede neural convolucional $(\mathrm{CNN})$ foi utilizado para investigar o desempenho diagnóstico para a diferenciação de massas hepáticas na tomografia computadorizada (TC) com contraste dinâmico. O estudo clínico retrospectivo utilizou conjuntos de imagens de TC de massas hepáticas ao longo de três fases (agente não-contrastado reforçado, arterial e retardado). As massas foram diagnosticadas de acordo com cinco categorias, desde cistos a carcinomas. $O$ treinamento supervisionado foi realizado usando 55.536 conjuntos de imagens obtidos em 2013. A CNN era composta por seis camadas convolucionais, três agrupamentos máximos e três camadas totalmente conectadas. Ela foi testada com 100 imagens de massa hepática obtidas em 2016. Os testes e treinamento foram realizados cinco vezes. A precisão para categorizar as massas hepáticas com o modelo $\mathrm{CNN}$ e a área sob a curva ROC para diferenciar as categorias $A B$ versus categorias $C E$ foram calculadas. $O$ estudo então concluiu que a aprendizagem profunda com CNN mostrou alto desempenho diagnóstico na diferenciação de massas hepáticas na TC dinâmica. ${ }^{27}$

Nicholas Bien e outros pesquisadores desenvolveram um modelo de rede neural de aprendizagem profunda a fim de detectar distúrbios em exames de ressonância magnética (RM) do joelho para melhorar a precisão do diagnóstico. O modelo previu, em questão de segundos, 3 resultados para os exames incluindo lesões meniscais e outros. O modelo ajudou, estatisticamente, médicos ortopedistas e radiologistas a diagnosticar exames de joelho, pois, usando conjuntos de dados internos e externos, geraram rapidamente classificações precisas de patologias clínicas de exames de RM de joelho. ${ }^{28}$

Pesquisadores da universidade de Stanford, na Califórnia, elaboraram um sistema usando algoritmos de "Deep Learning" para criar redes neurais convolucionais de aprendizagem profunda (CNN de aprendizagem profunda ou DNN) que pudessem analisar imagens clínicas e diagnosticar doenças de pele e comparar o seu desempenho no diagnóstico com o de médicos dermatologistas. Imagens inéditas de lesões comprovadas por biópsia foram exibidas e foi perguntado aos dermatologistas se eles iriam: Fazer a biopsia, tratar a lesão ou tranquilizar o paciente. A CNN foi treinada com base em 129.450 imagens clínicas credenciadas e comprovadas por biopsia, consistindo em 2.030 doenças diferentes. A CNN alcançou desempenho à altura de todos os especialistas testados em ambas as tarefas, demonstrando uma inteligência artificial capaz de classificar o câncer de pele com um nível de competência comparável aos dermatologistas. A CNN de aprendizagem profunda supera a média dos dermatologistas na classificação do câncer de pele utilizando imagens dermatoscópicas. Equipado com redes neurais profundas, 
dispositivos móveis podem potencialmente estender o alcance de dermatologistas fora do consultório. 16, 17, 29, 30, 31

O uso de redes neurais de aprendizagem profunda para classificação de imagens, detecção de objetos, segmentação, registro e outras tarefas, foi o foco de pesquisadores do Centro Médico da Universidade de Radboud, na Holanda. Na classificação de exames, normalmente tem uma ou várias imagens (um exame) servindo como modelo de entrada com uma única variável de diagnóstico como saída (por exemplo, doença presente ou não). A detecção de objetos de interesse ou lesões em imagens é uma parte fundamental do diagnóstico e é um dos trabalhos mais intensos para os médicos. Normalmente, as tarefas consistem na localização e identificação de pequenas lesões no espaço da imagem completa. Sistemas de detecção assistidas por computadores são projetados para identificar, automaticamente, lesões com alta precisão e diminuir o tempo de leitura dos especialistas humanos. ${ }^{25,30,31,32,33}$

\section{MACHINE LEARNING (ML) NO DIAGNÓSTICO POR IMAGEM DA COVID-19}

Uma pesquisa publicada por Mohamed Abd Elaziz et al. comprova que o ML tem demonstrado alto desempenho para várias aplicações de processamento de imagens como análise, classificação e segmentação. Foram utilizados vários métodos baseados em ML e DL para classificar radiografias de tórax de pacientes positivos para COVID-19 e pacientes negativos. Os autores propuseram um modelo CNN para o diagnóstico automático de COVID-19 a partir de imagens de radiografia de tórax. A precisão de classificação relatada é de $96,78 \%$ usando a arquitetura MobileNet. ${ }^{42}$

O método proposto por Mohamed et al. extrai as características de imagens de raios-X de tórax usando o software descritor FrMEMs, expoentes fracionais multicanais, empregando o algoritmo MRFODE que reduz/remove dados redundantes e/ou irrelevantes na pesquisa e utiliza um classificador de imagens conhecido como KNN que, por sua vez, irá identificar e mostrar imagens de interesse, após treinado. ${ }^{42}$ Uma ilustração do processo pode ser visualizada na figura 1 abaixo, onde é mostrado que no final do processo o classificador KNN traz o diagnóstico positivo e negativo para COVID-19. 


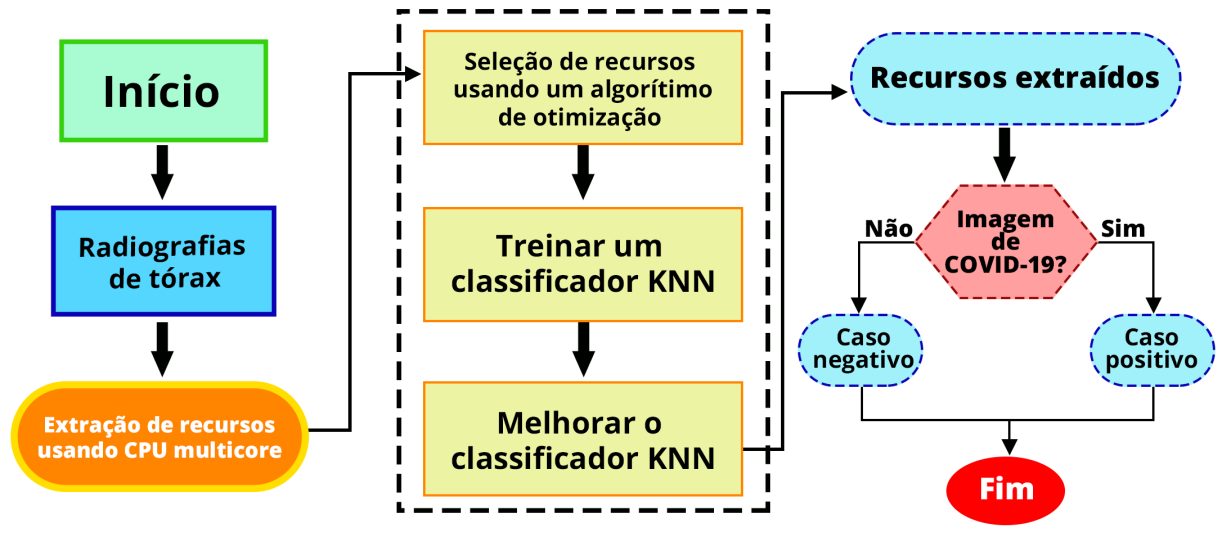

Figura 1. Fluxograma do método proposto pelos autores.

Fonte: Elaborado pelos autores.

Para realização do estudo, Mohamed et al. utilizou dois conjuntos de dados diferentes, onde:

O primeiro conjunto de dados coletados por Joseph Paul Cohen, Paul Morrison e Lan Dao no GitHub continham imagens extraídas de 43 publicações diferentes, com 216 imagens de COVID-19 positivas e 1.675 imagens COVID-19 negativas. ${ }^{42,43}$

Já o segundo conjunto de dados utilizados foram coletados de uma equipe de pesquisadores da Universidade do Qatar e da University de Dhaka, Bangladesh, junto com seus colaboradores do Paquistão e da Malásia em colaboração com médicos e adicionaram imagens da base de dados de COVID-19 da Sociedade Italiana de Radiologia Médica e Intervencionista (SIRM). Menciona-se que este segundo conjunto de dados se consistiu de 219 imagens de COVID-19 positivas e 1.341 imagens de COVID-19 negativas.

Menciona-se ainda que ambos os conjuntos de dados empregados são imagens do COVID-19 coletadas em pacientes com faixa etária entre 40 e 84 anos para ambos os sexos. ${ }^{42,44,45}$ 


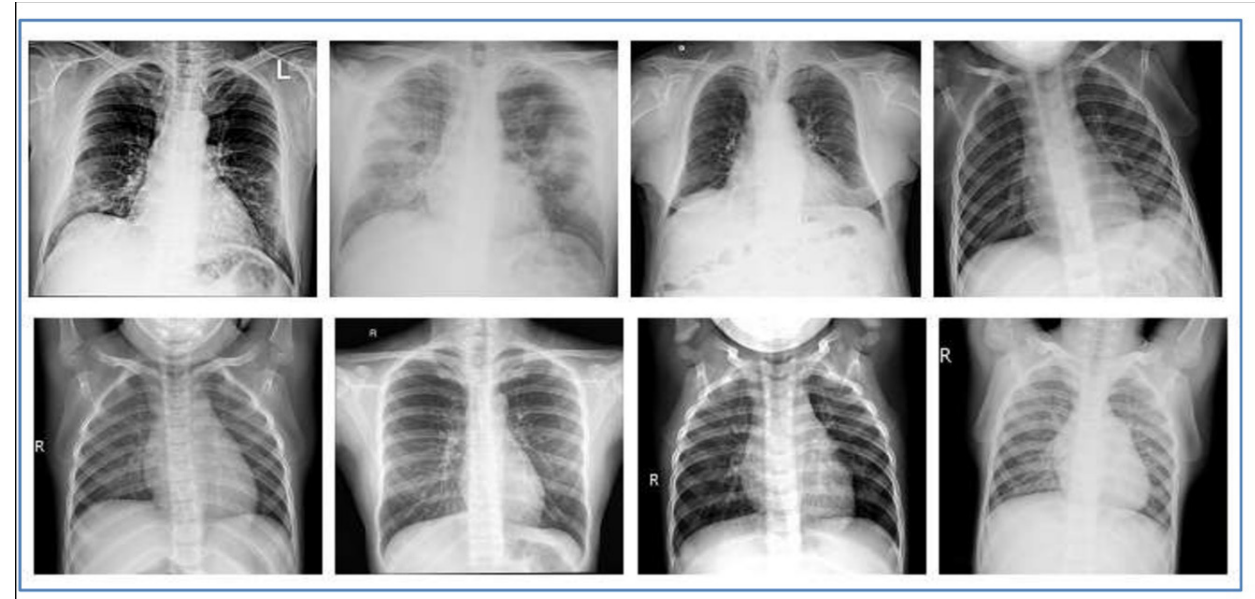

(a)

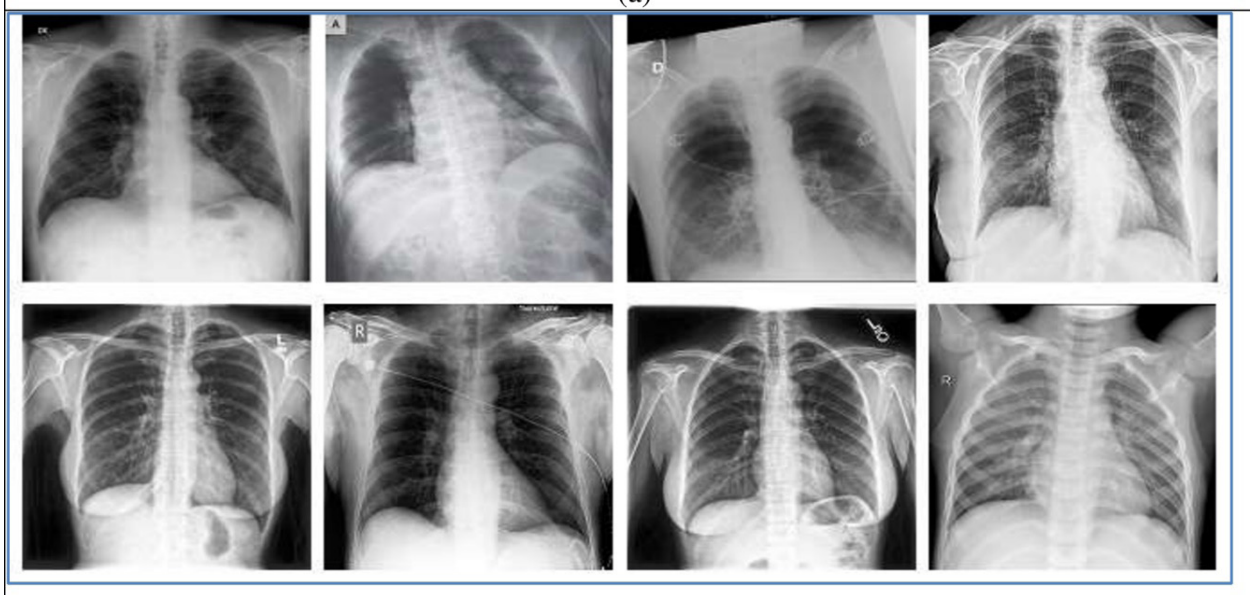

(b)

Figura 2. Amostra de imagens: (a) primeiro conjunto de dados; (b) segundo conjunto de dados.

Fonte: Elaziz MA et al. ${ }^{42}$

O método proposto por Mohamed et al. obteve alto desempenho nas métricas de exatidão, recall e avaliação de precisão com menor número de recursos quando comparados a outros métodos ${ }^{42}$. Menciona-se ainda que o direito do uso das imagens da Figura 2 foram cedidos pelos seus autores, durante esta pesquisa.

\section{MUDANÇAS E PREVISÕES}

Como exemplo mais específico do uso da IA para o diagnóstico por imagem, usando principalmente CNNs de aprendizagem profunda, a Google Inc. e a Google Brain (Equipe de pesquisa de inteligência artificial de aprendizagem profunda no Google), em parceria com a empresa Verily Life Sciences, desenvolveram uma inteligência artificial capaz de 
detectar metástases de câncer em imagens de patologia giga pixel, reduzindo a taxa de falso positivo para um quarto da taxa de um patologista. Os autores afirmam que esse método pode melhorar a precisão e consistência na avaliação de casos de câncer de mama e, potencialmente, melhorar os resultados dos pacientes. ${ }^{34}$

A Associação Canadense de Radiologistas - Canadian Association of Radiologists White Paper - prevê que a implementação da IA na radiologia durante a próxima década melhorará significativamente a qualidade, o valor e a profundidade da contribuição da radiologia para o atendimento ao paciente e para a saúde da população, e irá revolucionar os fluxos de trabalho dos radiologistas. ${ }^{6}$

A radiologia já tem experimentado grandes mudanças no mercado devido ao avanço tecnológico, e a inteligência artificial está cada vez mais em ascensão. Quanto mais curto for o tempo para laudar exames implica diretamente no aumento do tempo que o profissional terá para dar atenção e segmento ao diagnóstico do paciente, o que se torna um grande avanço na área saúde, pois quanto mais rápido um diagnóstico, mais chances podem ser encontradas para um tratamento precoce. Deste modo, cabe aos profissionais se empenharem em tentar entender o funcionamento da IA. 22, 33, 35, 36, 37

Este trabalho tem por objetivo verificar através de um minucioso estudo bibliográfico a atual presença da inteligência artificial aplicada à otimização do diagnóstico por imagem, mostrando sua eficiência em diversas tarefas, a fim de despertar os profissionais na área sobre a evolução da tecnologia para obtenção de um melhor e mais veloz diagnóstico médico, o que pode exigir uma adaptação dos mesmos para sua inserção ou permanência no mercado de trabalho.

\section{METODOLOGIA}

O trabalho desenvolvido seguiu os preceitos do estudo exploratório, por meio de uma pesquisa bibliográfica que, segundo Gil, é desenvolvida a partir de material já elaborado, constituído de livros e artigos científicos. ${ }^{38}$

Nesta perspectiva, a proposta de Gil (2008) foi utilizada nas seguintes etapas:

A seguir estão descritas as fontes que forneceram as respostas adequadas à solução do problema proposto:

Livros, divididos nas áreas de radiologia e informática, livros clínicos de radiologia e outros livros técnicos que abordam a temática, em idioma português e inglês, disponíveis na biblioteca da UNOESTE, publicados no período de 2010 a 2020.

Artigos científicos sobre a temática acessados nas bases de dados Scielo, Plos One, CONTER, MEDLINE, MINHA BIBLIOTECA, publicados nos últimos 10 anos (2010 a 2020).

Monografias disponíveis na biblioteca do curso de Radiologia da Unoeste publicadas no mesmo período. 
A coleta de dados seguiu a seguinte premissa:

A) Leitura exploratória de todo o material selecionado (leitura rápida que objetiva verificar se a obra consultada é de interesse para o trabalho); B) Leitura seletiva (leitura mais aprofundada das partes que realmente interessam); C) Registro das informações extraídas das fontes em instrumento específico (autores, ano, método, resultados e conclusões).

\section{RESULTADOS}

O objetivo desse estudo foi apresentar a presença e a importância da inteligência artificial aplicada na área médica de diagnóstico por imagem no cenário mundial, através de revisão bibliográfica atual.

Em geral, a IA tem mostrado resultados promissores quando se trata de análise diagnóstica comparado a profissionais humanos. A maioria dos testes comparativos da qualidade do diagnóstico entre a IA e radiologistas tem afirmado que ela tem capacidade igual ou um pouco maior na detecção de achados radiográficos, raramente perdendo para os profissionais quando se trata de análise de imagens.

A inteligência artificial tem acelerado o diagnóstico por imagem e tem sido amplamente utilizada para diagnósticos comparativos, extinguindo dúvidas diagnósticas.

\section{DISCUSSÃO E CONCLUSÃO}

Segundo a análise da pesquisa, a medicina está sendo impactada pela IA em três níveis: para os médicos, predominantemente através da rápida e precisa interpretação de imagem; para sistemas de saúde, melhorando o fluxo de trabalho e o potencial de redução de erros médicos; e para os pacientes, permitindo que eles processem seus próprios dados para promover a saúde. ${ }^{8,11,25}$

A evolução tecnológica no diagnóstico por imagem está acelerada. O conhecimento tecnológico se faz necessário para manusear os novos suportes (tanto hardware, quanto software) que estão sendo atualizados para otimizar os exames e diagnósticos.

Como a IA pode realizar, sozinha, tarefas de alta complexidade, é possível que haja uma revolução nos fluxos de trabalhos dos radiologistas. Essa revolução, por ser tecnológica, pode dificultar a permanência e determinar a entrada dos profissionais no mercado de trabalho.

Como Omir Antunes Paiva fala em seu artigo, os softwares irão fornecer dados que, geralmente, não são possíveis de serem extraídos por profissionais humanos, automaticamente priorizarão os exames de acordo com a gravidade, entre outros recursos, os quais podem ser citados, minimização de falhas nas imagens, reconstrução, melhoramento, diminuição de ruídos e artefatos das imagens adquiridas, que são corroborações de pesquisadores como Toru Higaki e Fang Liu. 22, 26, 35 
Todos os conceitos discutidos aqui são, também, complementados pelo Dr. Michael Forstin, o qual afirma que a inteligência artificial assumirá trabalhos corriqueiros como a determinação do tamanho ventricular após o aborto espontâneo, a medição das metástases hepáticas durante o tratamento, avaliação da idade óssea da mão e a medição dos ângulos na escoliose. Sendo que o necessário será apenas treinar os algoritmos para detectar com segurança o órgão alvo e a lesão. ${ }^{39}$

A Organização das Nações Unidas (ONU) observou que, quando o diagnóstico humano é acompanhado pela IA, a taxa de erro diminuiu para $0,5 \%$, contra os $3,5 \%$ dos médicos humanos. Estudos recentes também mostraram que a junção de clínicos humanos e a IA produz melhores resultados do que qualquer um deles isoladamente. ${ }^{40}$

Assim, é inegável os diversos benefícios advindos da inteligência artificial no diagnóstico por imagem por sua grande capacidade de processamento de imagens e reconhecimento de padrões. A evolução computacional, tanto do "software" quanto "hardwares" nas áreas médicas vem permitindo que os diagnósticos por imagem obtenham um enorme avanço qualitativo e quantitativo.

Desse modo, é evidente que a IA pode se tornar essencial para o diagnóstico por imagem e a necessidade de profissionais para as tarefas simples e rotineiras poderá entrar em declínio nos anos que se seguem, pelo fato de que as inteligências artificiais já estão sendo treinadas, corrigidas e melhoradas, e chegará o ponto em que todas essas atividades poderão, enfim, serem executadas sem a intervenção humana direta, revolucionando o setor de diagnóstico. ${ }^{41}$

No entanto, uma inteligência artificial que se preze precisa de uma grande base de dados e computadores com processadores potentes para um melhor funcionamento. Os algoritmos devem ser testados por muito tempo para que seja comprovada a sua excelência. Em resumo, os maquinários são caros e é preciso de muito tempo para testar e alimentar a IA com informações selecionadas.

Conclui-se que as atribuições da inteligência artificial, principalmente pelo uso das CNNs, deverão ajudar os radiologistas a alcançarem a excelência no diagnóstico médico. Esta área deverá se adaptar às inúmeras modificações que a IA possibilitará nos próximos anos, de modo a beneficiar o diagnóstico e cuidado dos pacientes. Portanto, os profissionais versados em radiologia que souberem usar a tecnologia a seu favor terão, claramente, vantagens em relação aos que não procuram se integrar a ela.

\section{AGRADECIMENTOS E CONFLITOS DE INTERESSE}

Agradeço aos meus queridos amigos Lara Antonia de Oliveira, Maiara da Silva Peloso e Rodrigo de Sousa Costa pela revisão de ortografia e gramática do meu trabalho e por todo o incentivo que me proporcionaram durante a minha pesquisa.

O autor declara não haver qualquer potencial conflito de interesse que possa 
interferir na imparcialidade deste trabalho científico.

\section{REFERÊNCIAS}

Al diagnostics need attention. Nature 2018;555:285. Doi: 10.1038/d41586-018-03067-x

Alves AF et al.; INTELIGÊNCIA ARTIFICAL: Conceitos, Aplicações e Linguagens. Rev. Conexão Eletrônica - Três Lagoas, MS - Volume 14 - Número 1 - Ano 2017.

Bengio Y, 2012. Practical recommendations for gradient-based training of deep architectures. In: Neural Networks: Tricks of the Trade. Springer Berlin Heidelberg, pp. 437-478.

Bengio Y, Lamblin P, Popovici D, Larochelle H, 2007. Greedy layer-wise training of deep networks. In: Advances in Neural Information Processing Systems. Université de Montréal. pp. 153-160. Disponível em: <http://papers.nips.cc/paper/3048-greedy-layer-wise-training-of-deep-networks.pdf>

Bien N, Rajpurkar P, Ball RL, Irvin J, Park AK, Jones E, et al. Deep-learning-assisted diagnosis for knee magnetic resonance imaging: Development and retrospective validation of MRNet. PLoS Med. 2018;15(11):e1002699. https://doi.org/10.1371/journal.pmed.1002699

Born, R.; Artificial Intelligence: The Case Against. Routledge, New York (2018)

Bradley JE.; Panagiotis, K.; Zeynettin, A.; Timothy, L. K.; Machine Learning for Medical Imaging. RadioGraphics. Local de publicação. v. 37. n. 2. p. 505-515. Fev. 2017. DOI < https://doi.org/10.1148/ rg.2017160130>

Charles EKJr.; Artificial Intelligence, Real Radiology. Publicado Online: 30 de janeiro de 2019. https:// doi.org/10.1148/ryai.2019184001

Chen MC, Ball RL, Yang $L$ et al.; Deep learning to classify radiology free-text reports. Radiology 286:845-852 (2018). https://doi.org/10.1148/radiol.2017171115

Chowdhury M. E., Rahman T., Khandakar A., Mazhar R., Kadir M. A., Mahbub Z. B. et al., "Can Al help in screening Viral and COVID-19 pneumonia? " arXiv preprint ar X iv:2003.13145, 2020.

Cohen J. P., Morrison P., and Dao L., "COVID-19 image data collection," arXiv preprint ar X iv:2003.11597, 2020.

D. A. L. Izzo Andrea. (2020, April-11-2020). Radiology. (2020). COVID-19 Database. Available: https:// www.sirm.org/category/senza-categoria/covid-19/

Elaziz MA, Hosny KM, Salah A, Darwish MM, Lu S, Sahlol AT (2020) New machine learning method for image-based diagnosis of COVID-19. PLoS ONE 15(6): e0235187. https://doi.org/10.1371/journal. pone. 0235187

Ertel W.; First-Order Predicate Logic. In Introduction to Artificial Intelligence; Springer: Berlin, Germany, 2017; 
Esteva A, Kuprel B, Novoa RA, Ko J, Swetter SM, Blau HM, Thrun S, 2017. Dermatologist-level classification of skin cancer with deep neural networks. Nature 542, 115-118.

Forsting M.; Artificial Intelligence with Radiology as a Trailblazer for Super-Diagnostics: An Essay. Fortschr Röntgenstr, 2019; 191(01): 73-78 DOI: 10.1055/a-0808-7772

Gil AC.; Métodos e Técnicas de Pesquisa Social. 6 ed. São Paulo: Atlas, 2008.

Gomes D.; Inteligência Artificial: Conceitos e Aplicações. Revista Olhar Científico - Faculdades Associadas de Ariquemes - V. 01, n.2, Ago./Dez. 2010. Disponível em: <http://www.olharcientifico. kinghost.net/index.php/olhar/article/view/49/37>

He K., Gkioxari G., Doll'ar P., Girshick R., Mar. 2017. Mask R-CNN. ArXiv e-prints. arXiv:1703.06870v3 [cs.CV] 24 Jan 2018.

Higaki T, Nakamura Y, Tatsugami F, Nakaura T, e Awai K.; Improvement of image quality at ct and mri using deep learning. Jpn. J. Radiol. 29 (2018), 1.

Hosny A, Parmar C, Quackenbush J, Schwartz LH, Aerts HJWL.; Artificial intelligence in radiology. Nat Rev Cancer, 2018. doi: 10.1038/s41568-018-0016-5.

HR Roth et al.; Deep learning and its application to medical image segmentation, Med. Imag. Technol., vol. 36, no. 2, pp. 63-71, Mar. 2018.

Litjens G, et al. 2017. A survey on deep learning in medical image analysis. Med. Image Anal. 42, 60-88. doi:10.1016/j.media.2017.07.005 Crossref,PubMed, Google Scholar

Liu F, Jang H, Kijowski R, Bradshaw T, Mcmillan AB. Deep Learning MR Imaging-based Attenuation Correction for PET/MR Imaging. Radiology 2018;286(2):676-684. pmid:28925823

Liu Y et al; 2017. Detecting cancer metastases on gigapixel pathology images. arXiv:1703.02442.

Loudon JS.; Detecting and Localizing Cell Nuclei in Medical Images. Norwegian University of Science and Technology: Julho de 2018.

Merriam - Webster definition of artificial intelligence. Acesso em: https://www.merriam-webster.com/ dictionary/artificial\%20intelligence Acessado em 14 de Junho de 2019.

Miyazaki C.; Redes neurais convolucionais para aprendizagem e reconhecimento de objetos 3D. Universidade de São Paulo. São Carlos, 2017.

Nóbrega Al. Radiologia Digital. 2002

O'shea K; Nash R.; An Introduction to Convolutional Neural Networks. Department of Computer Science, Aberystwyth University, Ceredigion; School of Computing and Communications, Lancaster University, Lancashire. arXiv:1511.08458v2 [cs.NE] 02 de Dezembro de 2015.

Osório F.; Bittencourt JR. Sistemas Inteligentes baseados em Redes Neurais Artificiais aplicados ao Processamento de Imagens. I WORKSHOP DE INTELIGÊNCIA ARTIFICIAL UNISC Universidade de Santa Cruz do Sul Departamento de Informática- Junho 2000. 
Paiva OA, Prevedello LM.; O potencial impacto da inteligência artificial na radiologia. Radiologia brasileira, 2017. DOI: http://dx.doi.org/10.1590/0100-3984.2017.50.5e1

Park SH, Han K.; Methodologic guide for evaluating clinical performance and effect of artificial intelligence technology for medical diagnosis and prediction. Radiology 286:800-809, 08 de Janeiro de 2018. https://doi.org/10.1148/radiol.2017171920

Park SH. Artificial intelligence in medicine: Beginner's guide. J Korean Soc Radiol 2018;78:301308. https://doi.org/10.3348/jksr.2018.78.5.301

Rajpurkar P, Irvin J, Ball RI, Zhu K, Yang B, Mehta H, et al. Deep learning for chest radiograph diagnosis: A retrospective comparison of CheXNeXt to practicing radiologists. PLoS Med. 2018;15(11):e1002686. https://doi.org/10.1371/journal.pmed.1002686

Tang A, et al.; Canadian association of radiologists white paper on artificial intelligence in radiology. Can. Assoc. Radiol. J. J. Assoc. Can. Radiol. 69, 120-135 (2018). https://doi.org/10.1016/j. carj.2018.02.002

Tilly Junior JG.; Física Radiológica. Rio de Janeiro. Guanabara Koogan, 2010.

Topol EJ.; High-performance medicine: The convergence of human and artificial intelligence. Nat. Med. 2019, 25, 44-56.

Vuong QH., Ho MT, Vuong TT, La VP, Ho MT, Nghiem KCP. et al.; Artificial Intelligence vs. Natural Stupidity: Evaluating Al readiness for the Vietnamese medical information system. Journal of Clinical Medicine. Journal of Clinical Medicine, 2019. https://doi.org/10.3390/jcm8020168

Yamashita R, Nishio M, Do RKG, Togashi K.; Convolutional neural networks: an overview and application in radiology. Insights Imaging. 2018 Jun 22; doi: 10.1007/s13244-018-0639-9

Yasaka K, Abe O.; (2018). Deep learning and artificial intelligence in radiology: Current applications and future directions. PLoS Med 15(11): e1002707. https://doi.org/10.1371/journal. pmed.1002707

Yasaka K, Akai H, Abe O, Kiryu S. Deep learning with convolutional neural network for differentiation of liver masses at dynamic contrast-enhanced CT: a preliminary study. Radiology 286:887-896 (2018). DOI: 10.1148/radiol.2017170706

Yu K, Kohane IS.; Framing the challenges of artificial intelligence in medicine. BMJ Quality \& Safety 2019;28:238-241. http://dx.doi.org/10.1136/bmjqs-2018-008551

Zech JR, Badgeley MA, Liu M, Costa AB, Titano JJ, Oermann EK.; Variable generalization performance of a deep learning model to detect pneumonia in chest radiographs: A crosssectional study. PLoS Med. 2018;15(11):e1002683. https://doi.org/10.1371/journal.pmed.1002683

Ziviani N.; A quarta revolução tecnológica. COMPUTAÇÃo COGNITIVA E A HUMANIZAÇÃO DAS MÁQUINAS - Fonte I Ed. 17 I Julho 2017 